\title{
INTERAÇÕES MEDICAMENTOSAS DE FITOTERÁPICOS UTILIZADOS NO TRATAMENTO DA INSÔNIA: UMA BREVE REVISÃO
}

\section{DRUG INTERACTIONS OF PHYTOTHERAPY USED IN THE INSOMNIA TREATMENT:}

\section{A BRIEF REVIEW}

\author{
Fabiana Sari Ferreira ${ }^{1}$
}

\begin{abstract}
1 - Mestre em Ciências Farmacêuticas, Universidade Estadual do Oeste do Paraná - UNIOESTE
\end{abstract} Autor para correspondência: sari.fabiana@gmail.com

\section{RESUMO:}

A insônia é um sintoma que pode ser definido pela dificuldade em iniciar, ou em manter o sono, sendo este uma atividade biológica essencial à sobrevivência do ser humano. $\mathrm{O}$ uso de fitoterápicos é uma alternativa ao tratamento. Assim, realizou-se uma revisão de literatura com o objetivo de investigar as interações medicamentosas causadas pelo uso dos fitoterápicos, comumente utilizados pela população brasileira no tratamento da insônia: Passiflora incarnata e a Valeriana officinalis. Ambos os fitoterápicos investigados neste trabalho apresentam importantes efeitos indesejáveis quando utilizados juntamente com outros medicamentos, fazendo-se indispensável o monitoramento farmacoterapêutico para que o tratamento seja bem-sucedido e sem prejuízos ao paciente.

Palavras-chave: Passiflora, Valeriana, Fitoterápicos.

\begin{abstract}
:
Insomnia is a clinical symptom defined as a difficulty for initiating or maintaining sleep. A good quality of sleep is essential to biological activity of the human body. The use of herbal medicines is an alternative to insomnia treatment; however, the indiscriminate use of these plant extracts can bring undesirable results. A literature review was carried out to investigate the drug interactions and adverse reactions caused by the phytotherapics: Passiflora incarnata and Valerian officinalis commonly used by Brazilian population for insomnia. Both phytotherapics investigated in this study have important undesirable effects when used in conjunction with other medicinal products. Thus, pharmacotherapeutic monitoring is essential to successful treatment and without harm to the patient.
\end{abstract}

Keywords: Passiflora, Valeriana, Phytotherapics.

\section{INTRODUÇÃO}

Apesar dos benefícios no uso de fitoterápicos, existe um grande número de notificações de eventos adversos no banco de dados da farmacovigilância da Agência Nacional de Vigilância Sanitária (ANVISA, 2018) referente a plantas medicinais ou seus derivados, confirmando a falsa crença que são inofensivos por serem produtos naturais.

De acordo com os dados da Organização Mundial da Saúde (OMS, 2003), 40\% dos brasileiros sofrem com dificuldade para dormir. Mas devido às dificuldades de acesso a 
consultas médicas para devido diagnóstico e tratamento, os fitoterápicos são um recurso utilizado por estes indivíduos, uso que na maioria das vezes é decorrente de automedicação e sem o acompanhamento de um profissional de saúde (ASSIS; JESUS, 2012; DUARTE et al., 2017).

A Passiflora incarnata e a Valeriana officinalis são os fitoterápicos mais utilizados como alternativa ao tratamento da insônia, que é um sintoma que pode ser definido pela dificuldade em iniciar, ou em manter o sono, e que necessita de diagnóstico específico para o tratamento ideal (OLIVEIRA; CERQUEIRA, 2016).

Por isso, esta pesquisa foi direcionada para investigar as interações medicamentosas e as reações adversas causadas pelo uso dos fitoterápicos que são comumente utilizados pela população brasileira com a finalidade de tratamento de distúrbios do sono.

\section{METODOLOGIA}

A pesquisa foi realizada com a finalidade exploratória, de caráter qualitativo e do tipo bibliográfica para obtenção de conhecimento geral e específico através de artigos científicos e web sites governamentais a fim de alcançar os objetivos desta pesquisa. A busca de informações e artigos científicos foi realizada nas bases de dados do Google Acadêmico e National Library of Medicine (PubMed).

Como critérios de inclusão, foram considerados artigos com resumos disponíveis online, artigos que abordam o assunto proposto, publicações preferencialmente nos últimos 10 anos, disponíveis nos idiomas de português ou inglês. Os critérios de exclusão foram os artigos que não abordam o tema em questão, sem resumo e de idiomas diversos aos requeridos. Para busca e seleção de artigos, foram utilizados os seguintes termos e suas associações: fitoterápicos, insônia, distúrbios do sono, interações medicamentosas, Valeriana e Passiflora.

\section{REFERENCIAL TEÓRICO}

\subsection{Insônia}

O sono é uma atividade biológica essencial para a sobrevivência do ser humano, sendo importante para a restauração do metabolismo cerebral, conservação da energia, 
e diversas outras funções. Apesar de o sono normal poder variar quanto à duração e seus estágios ao longo do desenvolvimento de cada pessoa, perturbações neste estado de repouso podem afetar de forma significativa as funções corporais (MÜLLER; GUIMARÃES, 2007).

A insônia é um sintoma que pode ser definido pela dificuldade em iniciar ou em manter o sono, ou ainda quando o sono não é reparador ou insuficiente para manter a qualidade de alerta durante o dia, prejudicando o bem-estar físico e mental e alterando o desempenho das tarefas diurnas (POYARES et al., 2003). Este sintoma afeta muito dos brasileiros, mas a prevalência é maior em pacientes do sexo feminino e também está associada a outros fatores de risco como o envelhecimento (SÁ; MOTTA; OLIVEIRA, 2007).

A anamnese do paciente é essencial para o diagnóstico, pois é necessário investigar os dados sobre os hábitos de sono, sejam eles noturnos ou diurnos. É importante identificar as possíveis presenças de causas clínicas, como depressão, ansiedade, pânico noturno e até mesmo averiguar o histórico do paciente com o uso de estimulantes, tranquilizantes, hipnóticos ou antidepressivos e também questionar o uso de álcool, tabagismo, bebidas com cafeína. Estes sintomas da insônia podem acontecer de forma isolada ou se manifestar juntamente com transtornos respiratórios, neurológicos e outros (POYARES et al., 2003).

Além da anamnese, a polissonografia é um exame laboratorial que se refere ao registro de algumas variáveis fisiológicas de forma simultânea durante o sono e após avaliação indica a presença de alterações da indução, da continuidade e da estrutura do sono, podendo auxiliar no reconhecimento dos sintomas dos distúrbios do sono (MONTI, 2000; SILVA, 1995).

Depois de avaliar o histórico e a gravidade de insônia do paciente, o terapeuta indica o formato de terapia mais apropriado. Visto que, este distúrbio do sono pode ser o sintoma de diversas doenças, o tratamento das enfermidades de base é indispensável, além disso, deve-se considerar a higiene do sono e se necessário o uso de medicamentos (POYARES et al., 2003).

O tratamento farmacológico é uma das alternativas para os pacientes que sofrem com insônia, sendo que os medicamentos sedativos-hipnóticos são os mais prescritos pelos médicos. Os hipnóticos promovem o tratamento sintomático da insônia e para o seu uso clínico deve ser avaliado o risco-benefício. Com propriedades hipnóticas, os benzodiazepínicos e barbitúricos são as substâncias de maior importância 
clínica (SERRA, 2006).

Os medicamentos da classe dos Hipnóticos Barbitúricos foram amplamente utilizados até o surgimento dos Hipnóticos Benzodiazepínicos, estes por sua vez apresentam quatro fundamentais atividades: ansiolítica, hipnótica, anticonvulsivante e relaxante muscular, o que faz deste grupo ter grande importância e ampla utilização na prática clínica. Uma vez que essas drogas são lipossolúveis, são capazes de atingir o Sistema Nervoso Central (SNC) e provocar alterações cognitivas e psicomotoras e por isso, ambas os fármacos apresentam importantes riscos, reações adversas, interações com outras drogas, além de dependência e tolerância (SILVA, 2010).

Muitos pacientes desconhecem os efeitos colaterais do uso em longo prazo desses medicamentos, por isso, uma alternativa ao tratamento com psicotrópicos é introdução de medicamentos fitoterápicos (fármacos obtidos exclusivamente por meio de vegetais) que causam uma leve ação sedativa e relaxante, induzindo ao sono, aliviando tensões e combatendo a agitação (ESTEVES, 2015).

\subsection{Fitoterápicos}

O tratamento de enfermidades baseado no uso de fitoterápicos eficazes para determinado fim chama-se fitoterapia. Segundo a legislação sanitária brasileira, RDC № 26 de 13 de maio de 2014, os medicamentos fitoterápicos são aqueles obtidos através do uso exclusivo de matérias-primas ativas vegetais, com a segurança e qualidade baseada em evidências clínicas (HARAGUCHI \& CARVALHO, 2010; ANVISA, 2014).

A grande diferença dos fitoterápicos para as plantas medicinais é que para usar as plantas medicinais é preciso conhecer, saber onde colher e como preparar a planta, a qual geralmente são utilizadas na forma de chás e infusões. No entanto, quando a planta medicinal é industrializada para a obtenção de um medicamento, temos o fitoterápico. Este processo de industrialização evita contaminações, padroniza a posologia e permite maior segurança de uso (ANVISA, 2018).

As terapias à base de produtos naturais utilizadas para o tratamento ou prevenção de doenças têm incluído estes produtos na pesquisa científica, por causa do fácil acesso e da crença de eventual diminuição dos efeitos colaterais. As origens de produtos que apresentam propriedades farmacologicamente ativas são das mais variadas e, as plantas, fazem parte dessa imensa fonte de recursos naturais (ALZOHAIRY, 2016).

No Brasil, os fitoterápicos a base de plantas popularmente conhecidas como: 
laranja amarga (Citrus vulgaris), valeriana (Valeriana officinalis), capim-limão (Andropogon citratus), erva-cidreira (Lantana geminata), camomila (Chamomilla recutita), melissa (Melissa bicornis), kava-kava (Piper methysticum) e maracujá (Passiflora incarnata) apresentam indicações de uso como sedativos, por isso poderiam auxiliar no caso de pacientes com insônia (ANVISA, 2011; TELESSAÚDE, 2016). No entanto, os medicamentos à base de extrato de Passiflora incarnata e Valeriana officinalis são utilizados com maior frequência (OLIVEIRA; CERQUEIRA, 2016).

Apesar de existir diferentes espécies, a Passiflora incarnata L. é a espécie com mais estudos farmacológicos e também é um fitoterápico comumente comercializado no Brasil. Popularmente conhecido como maracujá é amplamente utilizado devido a sua ação ansiolítica leve. Geralmente são empregadas as partes aéreas da planta para produção de extratos ou tinturas (ANVISA, 2008; GOSMANN et al., 2011).

A presença de compostos como os flavonoides é predominante nas amostras de $P$. incarnata e, de forma geral, os flavonoides são compostos comumente encontrados em diversos produtos naturais, protegendo esses organismos, principalmente, dos agentes oxidantes, desta forma, são apontados como um dos maiores grupos de metabólitos secundários (RODRIGUES DA SILVA et al., 2015).

Apesar do mecanismo de ação da Passiflora spp. ainda ser desconhecido, acreditase que podem estar envolvidos, a inibição da enzima monoamina oxidase (MAO) e a ativação dos receptores de ácido gama-aminobutírico (GABA) (KINRYS; COLEMAN; ROTHSTEIN, 2009).

A enzima MAO é geralmente encontrada em diversos tecidos e, no neurônio ela funciona para controlar qualquer excesso de neurotransmissores (noradrenalina, dopamina e serotonina) das vesículas sinápticas, portanto, quando a MAO esta inibida, pode ocorrer acúmulo de neurotransmissores e superestimulação dos receptores, podendo ser responsável pelos efeitos antidepressivos (GUYTON; HALL; GUYTON, 2006).

Já o GABA funciona como neurotransmissor inibitório principal no SNC dos mamíferos, reduzindo a excitabilidade neuronal por meio de vários tipos de mecanismos, o que influencia em muitas funções neuronais por estarem distribuídos de forma disseminada. Os fármacos moduladores dos receptores de GABA interferem na reatividade e na atenção, na formação da memória, na ansiedade, no sono e no tônus muscular (FORMAN et al., 2009).

A valeriana, como é conhecida popularmente (Valeriana officinalis L.) é uma planta herbácea muito utilizada desde os tempos dos gregos e romanos como sedativo leve, provocando diminuição do tempo para induzir o sono, menos movimentações ao dormir e 
ausência de ressaca matinal. Além disso, pode ser utilizada contra transtornos de ansiedade, angústia e também auxiliar em leves desequilíbrios do SNC (CUNHA, 2000; GRAVENA et al., 2009).

Esta planta é nativa da Europa e as suas raízes são as partes de maior interesse por apresentarem constituintes farmacológicos. Suas propriedades sedativa e ansiolítica são características provenientes dos compostos chamados de terpenoides, que são metabólitos secundários com ações no SNC, derivados de uma ou várias unidades isoprênicas, que são precursores dos demais compostos (GONÇALVES; MARTINS, 2006; OLIVEIRA; CERQUEIRA, 2016; PASSOS et al., 2009).

Vários mecanismos de ação têm sido sugeridos, entre eles está o ácido valerênico atuando no receptor GABA (CARDOSO et al., 2009). Devido aos efeitos inibitórios dos receptores GABA, como já descrito anteriormente, a excitabilidade neuronal vai ser diminuída e por isso são responsáveis pelo efeito hipnótico e ansiolítico desses fitoterápicos.

De modo geral, a valeriana tem sido um bom agente calmante por apresentar, sobretudo, um perfil farmacológico sem informações a respeito de efeitos colaterais quando utilizado nas doses recomendadas, e nem ter relatos de toxicidade aguda do seu extrato em estudos com animais (BISSOLI, 2013).

\section{CONCLUSÃO}

O levantamento bibliográfico foi realizado nos meses de junho e julho de 2018 através da plataforma Google Acadêmico, onde foram encontrados 190 artigos dos quais foram selecionados conforme título e resumo abordando o tema em questão. Após leitura na íntegra dos trabalhos com conteúdo correspondente a esta pesquisa, conforme necessidade, a complementação do conteúdo foi feita com os artigos encontrados na plataforma PubMed com busca livre, no entanto, apenas 11 compuseram este estudo.

\subsection{Possíveis Interações Medicamentosas}

Como os fitoterápicos não são produtos com um princípio ativo isolado, os medicamentos à base de $P$. incarnata possuem em sua composição outros compostos com frações alcaloídicas, derivados do indol, como harmana, harmina; e porções flavonoídicas, vitexina, isovitexina, dos quais podem provocar ações depressoras inespecíficas do SNC contribuindo para a ação sedativa e tranquilizante e ainda, podem interagir com outros medicamentos (NICOLETTI et al., 2007). 
Os fitoterápicos a base de $V$. officinalis também apresentam diversos metabólitos que fazem parte da composição desta planta além dos terpenoides, como os flavonoides, alcaloides e outros (GONÇALVES; MARTINS, 2006). E, devido à ação ansiolítica e sedativa destes ativos, a valeriana também pode interagir com outros compostos (ALEXANDRE; GARCIA; SIMÕES, 2005; NICOLETTI et al., 2007).

Como se pode perceber observando o QUADRO 1, a utilização dos fitoterápicos, tanto a base de $P$. incarnata, como de $V$. officinalis, juntamente com o uso de bebidas alcoólicas ou drogas, como a cocaína, pode acontecer de forma com que não programada, pois nem sempre o paciente tem o entendimento de que estas substâncias podem levar a efeitos colaterais quando interagem com outras drogas.

Além disso, é importante salientar que o paciente pode fazer uso de hipnóticos ou mesmo de inibidores da MAO para tratamento de ansiedade, depressão ou pânico e não correlacionar que estes medicamentos também influenciam nos distúrbios do sono e por isso pode fazer o uso concomitante desses fármacos com um dos fitoterápicos citados acima causando um aumento na intensidade da sonolência e podendo até provocar maior tempo de sedação.

QUADRO 1 - Principais interações medicamentosas descritas na literatura.

\begin{tabular}{|c|c|c|}
\hline Fitoterápico & Interações medicamentosas de maior importância & Efeitos \\
\hline \multirow{4}{*}{$\begin{array}{l}\text { Passiflora } \\
\text { incarnata L. }\end{array}$} & $\begin{array}{l}\text { Álcool } \\
\text { Benzodiazepínicos (Lorazepam ou Diazepam) } \\
\text { Barbitúricos (Fenobarbital) } \\
\text { Narcóticos (Codeína) }\end{array}$ & $\begin{array}{l}\text { Aumento da intensidade da } \\
\text { sonolência }\end{array}$ \\
\hline & $\begin{array}{l}\text { Inibidorers da enzima monoamina oxidase } \\
\text { (isocarboxazida, fenelzina e tranilcipromina) }\end{array}$ & Efeito aditivo \\
\hline & $\begin{array}{l}\text { Aspirina, varfarina, heparina } \\
\text { Antiplaquetários (clopidogrel) } \\
\text { Anti-inflmatórios não-esteroidais } \\
\text { (ibuprofeno e naproxeno) }\end{array}$ & Sangramento \\
\hline & Cafeína, guaraná ou efedra & Elevação da pressão arterial \\
\hline \multirow[t]{2}{*}{$\begin{array}{l}\text { Valeriana } \\
\text { officinalis }\end{array}$} & $\begin{array}{l}\text { Álcool } \\
\text { Benzodiazepínicos (Lorazepam ou Diazepam) } \\
\text { Barbitúricos (Fenobarbital) } \\
\text { Narcóticos (Codeína) } \\
\text { Anestésicos }\end{array}$ & $\begin{array}{l}\text { Aumento da intensidade } \\
\text { provocando maior tempo de } \\
\text { sedação }\end{array}$ \\
\hline & $\begin{array}{l}\text { Metronidazol } \\
\text { Dissulfiram }\end{array}$ & $\begin{array}{l}\text { Náuseas e vômitos } \\
\text { (quando } \quad V \text {. officinalis em } \\
\text { extrato alcoólico) }\end{array}$ \\
\hline
\end{tabular}

Fonte: Adaptado a partir de estudos realizados por ALEXANDRE; GARCIA; SIMÕES (2005); CARDOSO et al. (2009); NICOLETTI e colaboradores (2007); SALVI \& HEUSER (2008). 
O fitoterápico a base de $P$. incarnata quando administrado em conjunto com alguns anticoagulantes, antiplaquetários ou anti-inflamatórios pode levar a sangramento. Esta interação medicamentosa de extrema importância que deveria sempre ser orientada aos pacientes, pois é muito comum encontrar pessoas que usam indiscriminadamente antiinflamatórios como o naproxeno ou ibuprofeno para casos de analgesia ou febre e também, os idosos que frequentemente fazem uso de anticoagulantes em longo prazo.

Também é interessante orientar os pacientes, principalmente os mais jovens que fazem o uso de produtos estimulantes como cafeína ou guaraná para ganhar uma dose extra de energia ao longo do dia e, à noite se apresentarem dificuldades para dormir, fazem o uso de $P$. incarnata, podendo apresentar elevação da pressão arterial como consequência.

Resumindo, foi possível entender que o uso indiscriminado de medicamentos fitoterápicos pode levar a problemas de saúde. Além disso, cientificamente, sabe-se que todo medicamento de origem natural contém potenciais substâncias capazes de produzir os mais diversos tipos de ações e reações além dos descritos acima.

Estudos mostram que a Passiflora incarnata também pode causar diversos efeitos, sendo comumente observado dores de estômago, náuseas, sudorese e tontura além de efeitos mais raros como alteração do nível de consciência, flatulência, taquicardia e dispneia (MARJAN, 2010).

Também, existem registros de efeitos indesejáveis do uso do fitoterápico a base de Valeriana officinalis quando utilizado por longos períodos ou em doses elevadas, podendo ocorrer náuseas, midríase, diarreias, excitabilidade, cefaleias, vertigem e outros, desaparecendo quando o medicamento é suspenso (BISSOLI, 2013).

No Brasil, cada vez mais a população tem feito o uso de plantas medicinas e fitoterápicos em seus tratamentos. Através de um levantamento feito entre os anos de 2013 e 2015, a busca por produtos naturais aumentou cerca de $160 \%$, chegando a quase 16 mil pessoas que recebem esses produtos pelo Sistema Único de Saúde - SUS (PARANÁ, 2017).

Os resultados de um estudo realizado em população idosa mostraram também que, $64 \%$ das pessoas entrevistadas faziam uso de plantas medicinais e destes, $54 \%$ utilizavam junto algum medicamento sintético. Além disso, $17 \%$ dos entrevistados faziam uso de fitoterápico concomitantemente com outros medicamentos, evidenciando uma situação preocupante devido à frequentes interações medicamentosas (SILVA, 2016). 


\section{CONCLUSÃO}

Os fitoterápicos são medicamentos obtidos exclusivamente por matérias-primas vegetais que são importantes fontes de compostos biologicamente ativos, tendo grande importância e utilidade no tratamento da insônia e de diversas outras enfermidades. No entanto, os principais fitoterápicos utilizados no tratamento de distúrbios do sono, quando utilizados de forma indiscriminada, podem levar a consequências perigosas ao paciente e por isso devem ser motivo de preocupação.

Para que as interações medicamentosas e os efeitos adversos sejam evitados é necessário um trabalho interdisciplinar com os profissionais de saúde, diminuindo o aparecimento de problemas relacionados aos medicamentos e contribuindo para a saúde, bem-estar e qualidade de vida do paciente.

Ambos os fitoterápicos investigados neste trabalho apresentam importantes efeitos indesejáveis quando utilizados juntamente com outros medicamentos sem a devida atenção e orientação, fazendo-se indispensável o monitoramento farmacoterapêutico para que o tratamento seja bem-sucedido e sem prejuízo para o paciente.

\section{REFERÊNCIAS}

ALEXANDRE, R. F.; GARCIA, F. N.; SIMÕES, C. M. O. Fitoterapia baseada em evidências. Parte 1. Medicamentos fitoterápicos elaborados com ginkgo, Hipérico, Kava e Valeriana. Acta Farmaceutica Bonaerense, v. 24, n. 2, p. 300-309, 2005.

ALZOHAIRY, M. A. Therapeutics Role of Azadirachta indica (Neem) and Their Active Constituents in Diseases Prevention and Treatment. Evidence-Based Complementary and Alternative Medicine, v. 2016, p. 1-11, 2016.

ANVISA, A. N. DE V. S. INSTRUÇÃO NORMATIVA Nº 5, DE 11 DE DEZEMBRO DE 2008, 2008.

ANVISA, A. N. DE V. S. Formulário de Fitoterápicos Farmacopeia Brasileira, 2011.

ANVISA, A. N. DE V. S. RDC n²6 de 13 de maio de 2014. Registro de medicamentos fitoterápicos e o registro e a notificação de produtos tradicionais fitoterápicos., 2014. 
ANVISA, A. N. DE V. S. Medicamentos fitoterápicos e plantas medicinais. Disponível em: <http://portal.anvisa.gov.br/fitoterapicos>. Acesso em: 21 jun. 2018.

ASSIS, M. M. A.; JESUS, W. L. A. Access to health services: approaches, concepts, policies and analysis model. Ciencia \& Saude Coletiva, v. 17, n. 11, p. 2865-2875, 2012.

BISSOLI, J. R. Aspectos químicos e farmacológicos do medicamento fitoterápico Valeriana officinalis L. 2013.

CARDOSO, C. M. Z. et al. Elaboração de uma Cartilha Direcionada aos Profissionais da Área da Saúde, Contendo Informações sobre Interações Medicamentosas envolvendo Fitoterápicos e Alopáticos. Revista Fitos, v. 4, p. 56-69, 2009.

CUNHA, A. P. A Valeriana, planta medicinal de ontem e de hoje. Lisboa: 2000.

DUARTE, A. F. S. et al. O Uso De Plantas Medicinais Durante a Gravidez E Amamentação. Visão Acadêmica, v. 18, n. 4, p. 126-139, 2017.

ESTEVES, N. P. DE Q. Uso de Fitoterápicos como aliado no desmame do consumo inadequado de Benzodiazepínicos na atenção básica. Rio de Janeiro: Universidade do Estado do Rio de Janeiro, 2015.

FORMAN, S. A et al. Farmacologia da Neurotransmissão Gabaérgica e Glutamatérgica. 2. ed. Rio de Janeiro, 2009.

GONÇALVES, S.; MARTINS, A. P. Valeriana officinalis. Revista Lusófona de Ciências e Tecnologias da Saúde, v. 3, n. 2, p. 209-222, 2006.

GOSMANN, G. et al. Composição química e aspectos farmacológicos de espécies de Passiflora L. (Passifloraceae). Revista Brasileira de Biociências, v. 9, p. 88-99, 2011.

GRAVENA, R. A. et al. Uso da Valeriana officinalis em dietas de codornas japonesas na fase de postura. Biotemas, v. 22, n. 4, p. 185-191, 2009. 
GUYTON, A. C.; HALL, J. E.; GUYTON, A. C. Tratado de fisiologia médica. Elsevier, 2006.

HARAGUCHI, L. M. M.; CARVALHO, O. B. Plantas Medicinais. 2010.

KINRYS, G.; COLEMAN, E.; ROTHSTEIN, E. Natural remedies for anxiety disorders: potential use and clinical applications. Depression and Anxiety, v. 26, n. 3, p. 259-265, 2009.

MARJAN, I. E C. L. PASALIXSanto Amaro - SP, 2010.

MONTI, J. M. Insônia primária: diagnóstico diferencial e tratamento. Revista Brasileira de Psiquiatria, v. 22, n. 1, p. 31-34, 2000.

MÜLLER, M. R.; GUIMARÃES, S. S. Sleep disorders impact on daily functioning and life quality. Estudos de Psicologia, v. 24, n. 4, p. 519-528, 2007.

NICOLETTI, M. et al. Principais Interações no Uso de Medicamentos Fitoterápicos. Infarma, v. 19, n. 1, p. 32-40, 2007.

NICOLETTI, M. A. et al. Uso popular de medicamentos contendo drogas de origem vegetal e / ou plantas medicinais : principais interações decorrentes. Revista Saúde, v. 4, n. 1, p. 25-39, 2010.

OLIVEIRA, I. M. A.; CERQUEIRA, F. Fitoterapia: Interações medicamentosas e toxicidade na Farmácia de Oficina. 2016.

PARANÁ, S. DA S. Uso de fitoterápicos e plantas medicinais cresce no SUS., 2017. Disponível em: <http://portalms.saude.gov.br/noticias/agencia-saude/24205-uso-defitoterapicos-e-plantas-medicinais-cresce-no-sus>. Acesso em: 2 jun. 2018

PASSOS, C. S. et al. Terpenoides com atividade sobre o Sistema Nervoso Central (SNC). v. 19, n. 1A, p. 140-149, 2009. 
POYARES, D. et al. I Consenso Brasileiro de Insonia. Hypnos: rev sono, v. 4, n. (Supl 2), p. 5-39, 2003.

RODRIGUES DA SILVA, L. et al. Flavonóides: constituição química, ações medicinais e potencial tóxico. Acta toxicológica argentina, v. 23, p. 36-43, 2015.

SÁ, R. M. B. DE; MOTTA, L. B. DA; OLIVEIRA, F. J. DE. Insomnia: prevalence and related risk factors in elderly outpatients. Rev. bras. geriatr. gerontol, v. 10, n. 2, p. 217-230, 2007.

SALVI, R. M.; HEUSER, E. D. Interações: medicamentos x fitoterápicos : em busca de uma prescrição racional. 2008.

SERRA, J. Terapêutica farmacológica da insónia. Revista Portuguesa de Clínica Geral, v. 22, p. 625-632, 2006.

SILVA, R. S. DA. Introduçao a Tecnica de Polissonografia. Brazilian Journal of Epilepsy and Neurophysiology, v. 1, n. 1, p. 23-32, 1995.

SILVA, N. C. S. Tudo que é natural não faz mal? Investigação sobre o uso de Plantas Medicinais e Medicamentos Fitoterápicos por idosos , na cidade de lapu - Leste de Minas Gerais. Unica Cadernos Acadêmicos, v. 2, n. 1, 2016.

SILVA, P. Farmacologia. 8. ed. 2010.

TELESSAÚDE, S. C. Fitoterapia para insônia. Florianópolis: 2016. Disponível em: <https://repositorio.ufsc.br/xmlui/handle/123456789/169257>. Acesso em: 24 jun. 2018. 